\title{
Intravenous calcium as a pressor in a swine model of hypoxic pseudo-pulseless electrical mechanical activity-a preliminary report
}

Alexander L. Lindqwister ${ }^{1}$, Joshua W. Lampe ${ }^{2}$, Jeffrey R. Gould², Christopher L. Kaufman², Karen L. Moodie and Norman A. Paradis ${ }^{1,3^{*}}$

\section{* Correspondence: Norman.A.}

Paradis@hitchcock.org

${ }^{1}$ Geisel School of Medicine

Dartmouth College, Hanover, $\mathrm{NH}$

03775, USA

${ }^{3}$ Geisel School of Medicine,

Dartmouth Hitchcock Medical

Center, 1 Medical Center Dr \#4B,

Lebanon, NH 03756, USA

Full list of author information is

available at the end of the article

\begin{abstract}
Background: Pseudo-pulseless electrical activity (pseudo-PEA) is a lifeless form of profound cardiac shock characterized by measurable cardiac mechanical activity without clinically detectable pulses. Pseudo-PEA may constitute up to $40 \%$ of reported cases of cardiac arrest. Resuscitation from pseudo-PEA is often associated with hypotension refractory to catecholamine pressors. We hypothesized that this post-resuscitation state may be associated with hypocalcemic hypotension responsive to intravenous calcium.
\end{abstract}

Methods: Using pre-existing data from our hypoxic swine pseudo-PEA model, we measured blood pressure, hemodynamics, and electrolytes. Physiological data were analyzed on a heartbeat by heartbeat basis. The midpoint of the calcium response was defined using change of curvature feature detection. Hemodynamic parameters were shifted such that the value at the midpoint was equal to zero.

Results: In 9 animals with refractory hypotension, we administered 37 boluses of intravenous calcium in the dosage range of 5-20 mg. Comparisons were made between the average values in the time period $40-37 \mathrm{~s}$ before the midpoint and 35$40 \mathrm{~s}$ after the midpoint. Of the 37 administered boluses, 34 manifested a change in the blood pressure, with mean aortic pressure, systolic and diastolic pressures all increasing post bolus administration.

Conclusions: Administration of intravenous calcium may be associated with a pressor-like response in refractory hypotension after resuscitation from pseudo-PEA. Relative ionized hypocalcemia may cause hypotension after resuscitation from pseudo-PEA. Therapy with intravenous calcium should be further investigated in this setting.

Keywords: Pressor, Calcium, PEA, Pulseless electrical activity
(-) The Author(s). 2020 Open Access This article is licensed under a Creative Commons Attribution 4.0 International License, which permits use, sharing, adaptation, distribution and reproduction in any medium or format, as long as you give appropriate credit to the original author(s) and the source, provide a link to the Creative Commons licence, and indicate if changes were made. The images or other third party material in this article are included in the article's Creative Commons licence, unless indicated otherwise in a credit line to the material. If material is not included in the article's Creative Commons licence and your intended use is not permitted by statutory regulation or exceeds the permitted use, you will need to obtain permission directly from the copyright holder. To view a copy of this licence, visit http://creativecommons.org/licenses/by/4.0/. 


\section{Introduction}

The post-resuscitation state is often characterized by hypotension that is refractory to pressors [1]. While it is likely that the myocardial and vasomotor processes are multifactorial, it has been proposed that disordered calcium homeostasis is a central factor [2, 3], as ionized calcium plays an important role in both inotropy and vasomotor tone [4].

Nieman et al. previously reported that ionized hypocalcemia occurs post return of spontaneous circulation (ROSC) in their swine model of ventricular fibrillation [2, 3], and that administration of intravenous (IV) calcium gluconate is associated with a transient improvement in hemodynamics.

Historically, clinical cardiac arrest has been classified into two broad categories that include ventricular fibrillation and pulseless electrical activity (PEA). The latter category was first called electromechanical dissociation (EMD), but underwent a name change when studies revealed that in a substantial fraction of patients, pulse pressures and ventricular mechanical function were detectible $[5,6]$. The detection of pulses with advanced technology renders PEA an imprecise descriptor, and we now refer to the cardiovascular state of subjective clinical cardiac arrest with detectable hemodynamics as "pseudo-PEA." Specifically, pseudo-PEA is a global hypotensive ischemic state with retained coordinated myocardial contractile activity and an organized ECG.

Similar to ventricular fibrillation, pseudo-PEA causes a profound global ischemia and is often associated with post-resuscitation hypotension [7]. Extending Nieman's report of IV calcium responsive hypotension after resuscitation from fibrillation cardiac arrest, we now provide a preliminary report of a calcium pressor response in hypotension after resuscitation from pseudo-PEA.

\section{Methods}

The manuscript contains a post hoc analysis of data originally obtained in related studies using our established pseudo-PEA model $[8,9]$. These studies were conducted in accordance with the guidelines of the National Research Council of the National Academies and with the approval of the Dartmouth College Institutional Animal Care and Use Committee. The model utilized was a variation on previously described porcine asphyxial P-EMD preparation $[5,6,10]$.

\section{Surgical prep}

Three cohorts $(N=38)$ of domestic farm raised Yorkshire swine weighing approximately $30 \mathrm{~kg}$ were fasted overnight with free access to water and then sedated with an intramuscular injection of ketamine $(30 \mathrm{mg} / \mathrm{kg})$. After endotracheal intubation, anesthesia was initiated and maintained with isoflurane (0.5-4\%) and oxygen (1-3 L/ min). During preparation, ventilation was provided by a volume-controlled ventilator (GE Datex-Ohmeda Modulus SE, Madison, WI) with $100 \% \mathrm{O}_{2}$ (tidal volume of 15-20 $\mathrm{cc} / \mathrm{kg}$ and ventilation rate of $8-15$ breaths per minute) during initiation, reducing to $30 \%$ shortly thereafter. Ventilation rate and tidal volume were initially adjusted to maintain normocapnia (the end-expiratory partial pressure of $\mathrm{CO}_{2}$ between 35-45 $\mathrm{mmHg}$ ) as measured continuously by a capnometer (CO2SMO, Novametrix, Wallingford, CT) placed in the airway. Arterial blood gases (I-Stat, Abbott Point of Care, Princeton, NJ) were analyzed to confirm adequate baseline ventilation. Throughout the 
experiment, the animals were monitored using ECG, end-tidal $\mathrm{CO}_{2}$, and arterial blood pressure. In addition, depth of anesthesia was continuously assessed.

The animals were secured in a supine position and were given normal saline at a rate of $10 \mathrm{ml} / \mathrm{kg}$ per hour through a vein to maintain a central venous pressure of $\sim 5$ mmHg. Through either ultrasound-guided percutaneous cannulations or surgical cutdown, micromanometer catheters with a lumen were placed into (1) the right atrium via the femoral vein, and (2) the descending aorta through the femoral artery for pressure measurements. Cohort 1 utilized Tru-Wave Pressure Transducers (Edwards Lifesciences, Irvine, CA), and cohorts 2 and 3 utilized SPR-350 (Millar Instruments, Houston, TX). All catheters were positioned under fluoroscopic guidance, and unfractionated heparin (100 units $/ \mathrm{kg}$ ) was given to prevent catheter clotting. Flow probes were placed around the carotid artery (3 PS probe, Transonic, Ithaca, NY) and jugular vein (2.5 PS probe, Transonic, Ithaca, NY) via a cut-down procedure.

After instrumentation, baseline measurements were obtained for all variables including blood gas analyses. Analog outputs of the physiological parameters were digitized and stored in data files on a personal computer for further analysis using a 16-channel computerized data-acquisition system at a sampling rate of $1000 \mathrm{~Hz}$ (Powerlab 16SP, ADInstruments, Castle Hill, Australia). Raw data channels included ECG, aortic pressure, right atrial pressure, intra-cranial pressure, capnography, carotid blood flow, and jugular blood flow.

\section{Pseudo-PEA induction and chest compression}

Animals were converted to continuous intravenous anesthesia using ketamine $(50 \mathrm{mcg} /$ $\mathrm{kg} / \mathrm{min})$ and fentanyl $(0.45 \mathrm{mcg} / \mathrm{kg} / \mathrm{min})$, isoflurane was gradually discontinued. Depth of anesthesia was ensured via monitoring blood pressure, heart rate, and jaw tone. The IV anesthesia protocol was maintained $15 \mathrm{~min}$ to allow isoflurane washout and to establish a stable level of continuous IV anesthesia prior to initiation of the hypoxia protocol.

Arterial blood gases were measured at baseline and after each episode of pseudo-PEA (i-STAT, Abbott Point of Care, Abbott Park, IL). Measurements included: $\mathrm{pH}, \mathrm{pCO}$, $\mathrm{pO} 2$, base excess, $\mathrm{HCO} 3, \mathrm{TCO} 2, \mathrm{O} 2$ percent saturation, $\mathrm{Na}(\mathrm{mmol} / \mathrm{L}), \mathrm{K}(\mathrm{mmol} / \mathrm{L})$, ionized calcium (iCa), glucose, hematocrit, and hemoglobin.

Once adequate anesthesia has been confirmed, the animals were paralyzed using vecuronium $(1.0 \mathrm{mg} / \mathrm{kg})$ to minimize gasping [11]. Baseline data were measured before any injury occurred. Round 1 data were measured after resuscitation from the first pseudo-PEA injury. Data were also measured after resuscitation from subsequent pseudo-PEA injuries. The number of injuries per animal is variable, but the maximum number of hypoxic episodes is 5 . Animals were ventilated with a progressively hypoxic gas mixture of $\mathrm{O}_{2} / \mathrm{N}_{2}$. Gas concentrations were measured using an oxygen concentration analyzer (Oxygen Analyzer S-3A/II, Applied Electrochemistry, VMETEK) and the concentration of $\mathrm{O} 2$ was decreased until pseudo-PEA was achieved. Onset of pseudoPEA was defined as sustained aortic systolic pressure $\leq 50 \mathrm{mmHg}$ recorded by the aortic catheter in the presence of an organized cardiac rhythm.

Animals were treated with mechanical chest compressions delivered at a depth of 5 $\mathrm{cm}$ and at a variable rate. Compressions were delivered for a total of $6 \mathrm{~min}$. After $6 \mathrm{~min}$ 
of chest compressions, the $\mathrm{FiO} 2$ was set to $100 \%$, and chest compressions were continued until ROSC was achieved. ROSC was defined as a systolic pressure $>60 \mathrm{mmHg}$ without chest compressions. If ROSC was detected at any point during the chest compressions with ongoing hypoxia, compressions were terminated and $\mathrm{FiO} 2$ was set to $100 \%$.

\section{Post resuscitation treatment}

Arterial blood gases were measured $10 \mathrm{~min}$ after ROSC in all animals and for all episodes of pseudo-PEA. Three related experiments that utilized the same method of hypoxic injury and resuscitation were performed on three separate cohorts of swine (Tables 1 and 2). Animals in cohorts 1 and 2 did not receive ionized calcium. The observation that ionic calcium concentration decreases following pseudo-PEA led to the addition of $\mathrm{Ca}$ gluconate to the treatment regimen post-resuscitation for the 14 animals in cohort 3. Animals in cohort 3 were administered ionized calcium in an ad hoc fashion during the recovery phase when MAP was not sufficient or stable. A bolus of $\mathrm{Ca}$ gluconate would be delivered intravenously if the aortic blood pressure was decreasing after resuscitation. This meant that calcium might be given to treat blood pressures before the blood chemistries were drawn, resulting in some missing data.

Table 1 Results of cohorts 1 and 2 of animal experiments

\begin{tabular}{|c|c|c|c|c|c|c|c|c|c|c|c|c|c|}
\hline & \multirow[t]{2}{*}{ Animal } & \multicolumn{2}{|c|}{ Baseline } & \multicolumn{2}{|c|}{ Round 1} & \multicolumn{2}{|c|}{ Round 2} & \multicolumn{2}{|c|}{ Round 3} & \multicolumn{2}{|c|}{ Round 4} & \multicolumn{2}{|c|}{ Round 5} \\
\hline & & $\overline{\mathrm{pH}}$ & $\overline{\mathrm{iCa}}$ & $\overline{\mathrm{pH}}$ & $\overline{\mathrm{iCa}}$ & $\overline{\mathrm{pH}}$ & $\overline{\mathrm{iCa}}$ & $\overline{\mathrm{pH}}$ & $\overline{\mathrm{iCa}}$ & $\mathrm{pH}$ & $\overline{\mathrm{iCa}}$ & $\overline{\mathrm{pH}}$ & $\overline{\mathrm{iCa}}$ \\
\hline \multirow[t]{14}{*}{ Cohort 1} & 1 & 7.46 & 1.29 & 7.29 & 1.21 & 7.32 & 1.12 & 7.31 & 1.1 & 7.28 & 1.17 & 7.29 & 1.20 \\
\hline & 2 & 7.50 & 1.26 & 7.27 & 1.21 & 7.23 & 1.24 & 7.37 & 1.23 & 7.34 & 1.28 & 7.35 & 1.26 \\
\hline & 3 & 7.47 & 1.28 & 7.32 & 1.24 & 7.32 & 1.25 & 7.29 & 1.24 & 7.32 & 1.27 & 7.4 & 1.25 \\
\hline & 4 & 7.49 & 1.29 & 7.25 & 1.20 & 7.20 & 1.25 & 7.24 & 1.18 & 7.29 & 1.15 & 7.24 & 1.17 \\
\hline & 5 & 7.50 & 1.35 & 7.53 & 1.33 & 7.48 & 1.26 & 7.51 & 1.26 & 7.49 & 1.25 & 7.31 & 1.25 \\
\hline & 6 & 7.49 & 1.36 & 7.46 & 1.25 & 7.46 & 1.22 & 7.39 & 1.25 & 7.36 & 1.28 & 7.42 & 1.72 \\
\hline & 7 & 7.48 & 1.39 & 7.51 & 1.34 & 7.48 & 1.33 & 7.51 & 1.30 & 7.50 & 1.28 & 7.50 & 1.28 \\
\hline & 8 & 7.51 & 1.41 & 7.33 & 1.29 & & & & & & & & \\
\hline & 9 & 7.50 & 1.40 & 7.40 & 1.07 & 7.44 & 1.27 & 7.45 & 1.28 & 7.38 & 1.28 & & \\
\hline & 10 & 7.47 & 1.36 & 7.09 & 1.28 & & & & & & & & \\
\hline & 11 & 7.48 & 1.33 & 7.24 & 1.32 & 7.27 & 1.25 & & & & & & \\
\hline & 12 & 7.50 & 1.17 & 7.33 & 1.27 & 7.33 & 1.27 & & & & & & \\
\hline & 13 & 7.48 & 1.29 & 7.33 & 1.25 & 7.38 & 1.22 & & & & & & \\
\hline & 14 & 7.48 & 1.30 & 7.33 & 1.32 & & & & & & & & \\
\hline \multirow[t]{10}{*}{ Cohort 2} & 1 & 7.24 & 1.19 & & & & & & & & & & \\
\hline & 2 & 7.23 & 1.20 & & & & & & & & & & \\
\hline & 3 & 7.49 & 1.23 & 7.32 & 1.14 & & & & & & & & \\
\hline & 4 & 7.48 & 1.29 & 7.48 & 1.29 & & & & & & & & \\
\hline & 5 & 7.51 & 1.13 & & & & & & & & & & \\
\hline & 6 & 7.43 & 1.17 & & & & & & & & & & \\
\hline & 7 & 7.45 & 1.33 & 7.25 & 1.21 & 7.33 & 1.11 & 7.31 & 1.16 & & & & \\
\hline & 8 & 7.42 & 1.07 & 7.23 & 1.07 & 7.28 & 0.92 & 7.28 & 0.88 & & & & \\
\hline & 9 & 7.48 & 1.34 & 7.34 & 1.17 & 7.33 & 1.11 & 7.32 & 1.11 & 7.34 & 1.08 & & \\
\hline & 10 & 7.45 & 1.23 & 7.32 & 1.11 & 7.20 & 0.92 & 7.20 & 1.06 & & & & \\
\hline
\end{tabular}




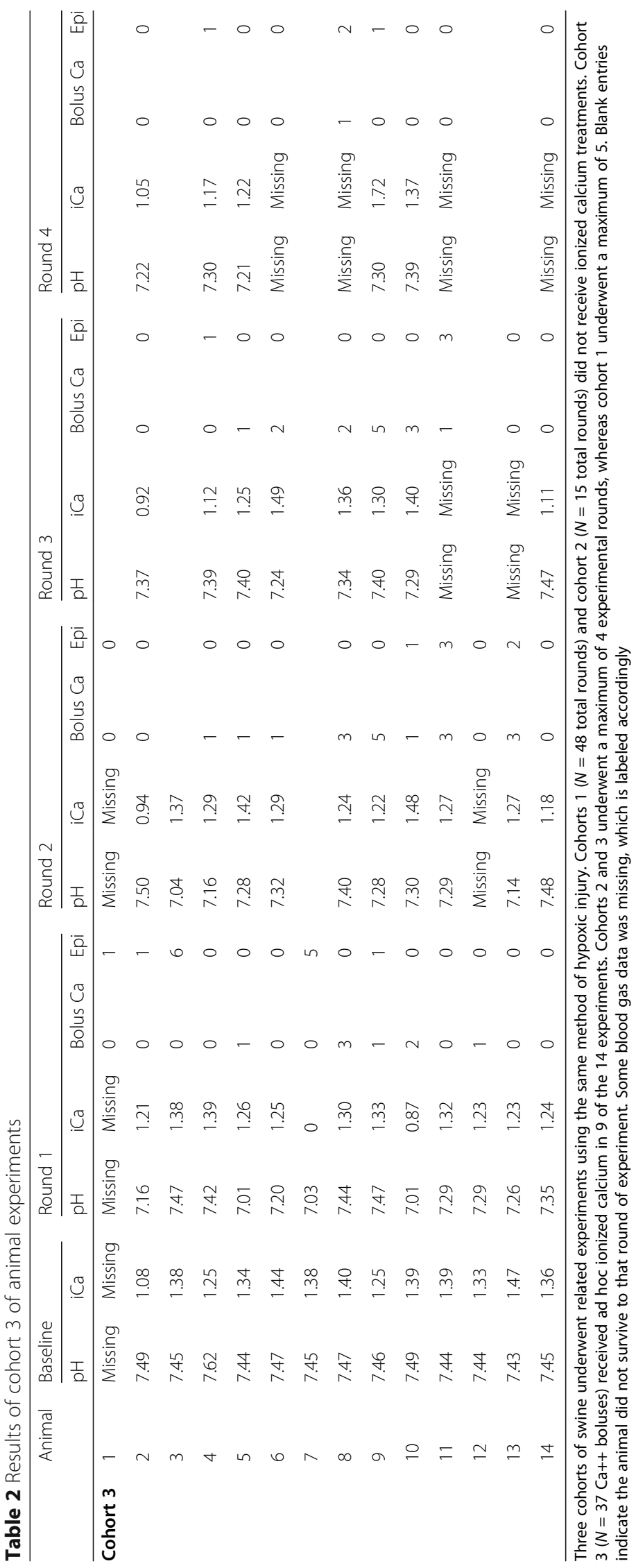


In addition, epinephrine and sodium bicarbonate were also delivered to support perfusion pressures and correct post-resuscitation blood chemistry as needed. As a result, different animals received different numbers and dosages of calcium boluses. Individual animals received as many as five periods of hypoxia-induced pseudo-PEA followed by resuscitation. Time of delivery of epinephrine, bicarbonate, and calcium were annotated in the physiological data file from each experiment.

\section{Data selection}

Of the 14 animals in cohort 3, 9 received calcium injections, for a total of 37 boluses of calcium. For each recorded calcium bolus, a data subset was exported that included time intervals before and after the bolus. Because the calcium was delivered in an unprotocolized manner, the amount of time before (or after) the bolus varied for each calcium injection. In the event that a calcium injection was preceded or followed by a different injection of calcium or injections of bicarbonate or epinephrine, the data subset started (or ended) half-way between the two injections. This was done so as to isolate the effect of the injection of calcium from the effects of other injections. One critical limitation of this method is that there was significant variation in the amount of time before and after a calcium injection. The minimum amounts of time before and after each calcium injection was $40 \mathrm{~s}$ and our analysis focused on these intervals.

\section{Data analysis}

Individual heartbeats were identified using a python script (Anaconda 1.9.6, Spyder version 3.1.2, Pandas version 0.22.0, Numpy version 1.14.0). Once the location of each heartbeat was identified, the mean arterial pressure (MAP), the systolic aortic pressure (AoS), and the diastolic aortic pressure (AoD) were all calculated using normal conventions. The effect of calcium on hemodynamics was clearly observable in most cases and the physiological effect could be approximated using a sigmoid shape. However, the heterogeneity of the mean blood pressures and their slope during recovery from pseudo-PEA would have obscured any analysis of the raw data. To understand the size of the pressor effect of calcium, blood pressure data were normalized such that the midpoint of observed effect occurred at a pressure of $0 \mathrm{mmHg}$ and a time of $0 \mathrm{~s}$. This normalization was performed by a second python script which used the zero-crossing point in the second derivative of the systolic aortic pressure, the second derivative of the coronary perfusion pressure, and the second derivative of the cerebral perfusion pressure to approximate the midpoint of the sigmoid response to a calcium bolus. Once the midpoint was found, pressures were normalized by subtracting the pressure value at the midpoint, and time was normalized by subtraction of the time when the midpoint occurred. Once the MAP, AoS, and AoD pressures were normalized, the mean values and standard deviations were calculated for the times $-40 \mathrm{~s}<t<40 \mathrm{~s}$.

\section{Statistical methods}

The normality of the mean aortic pressure, systolic aortic pressure, and diastolic aortic pressure signals was verified using the Shapiro-Wilk test. The hemodynamics were compared from the time period $-40<t<-36$ (before) and the time period $36<t<40$ (after), where $t$ represents the time normalized to the effect of IV administration of 
calcium. Measurements were averaged across these two $4 \mathrm{~s}$ periods and compared via a paired $t$ test (STATA $\mathrm{v}$ 15.1). The serum baseline, post-round 1 , and post $2+$ round ionic calcium concentrations were aggregated between cohorts 1 and 2 . The normality of the aggregate initial $\mathrm{iCa}$, post-round $1 \mathrm{iCa}$, and average remaining post $2+$ rounds $\mathrm{iCa}$ was verified using the Shapiro-Wilk test. These measurements were compared via one-way ANOVA (Excel v. 16.38).

\section{Results}

Tables 1 and 2 demonstrate the blood chemistry results for all pigs from each cohort. Blank entries indicate that the animal did not survive to that round, whereas "missing" indicates incomplete or missing data. One-way ANOVA analysis of the aggregate data for animals who did not receive bolus calcium (cohorts 1 and 2, Table 3) shows significant decreases in baseline, post-round 1 , and subsequent rounds iCa $(p=0.03)$. In cohort 1 , some animals did not require CPR for resuscitation, however, there was no significant difference in iCa between pigs who did or did not require CPR ( $p=0.89)$. Animals in cohorts 2 and 3, all received CPR.

The blood chemistries of animals that received IV calcium are shown in Table 4 as a function of the experimental round. Because of the calcium treatments, measured $\mathrm{iCa}$ values were not different between the rounds. Base excess (BE), bicarbonate (HCO3), and total carbon dioxide (TCO2) were lower after PEA than at baseline but were not affected by subsequent pseudo-PEA injuries.

Calcium doses were delivered in the range of 5-20 mg with the majority of the doses being $10 \mathrm{mg}$. In total, 37 unique boluses of calcium were annotated in the experimental record. The normalized aortic blood pressures from all 37 boluses are shown in Fig. 1. The data in this figure have been smoothed to reduce the ventilation artifact so that the individual responses are easier to discern. The different responses in Fig. 1 demonstrate the hyperdynamic nature of the hemodynamics after resuscitation from pseudoPEA. The effect of the calcium bolus is observed before time 0 because time 0 was set to the midpoint of the pressor effect. Because the amount of data exported for each delivered calcium bolus depended on the time interval between the calcium bolus of interest and the injection of other medications such as epinephrine, bicarbonate, and other doses of calcium, the different lengths of the data segments also demonstrate the variability in the amount of hemodynamic support the animals needed after resuscitation.

To compare the hemodynamics before and after the calcium bolus, we chose timespan of $[-40 \mathrm{~s}<t<40 \mathrm{~s}]$ for which data are present in all animals. Mean aortic pressures (MAP), systolic aortic pressure (AoS), and diastolic aortic pressure (AoD) were

Table 3 Results of blood chemistries from cohorts 1 and 2 that did not receive calcium treatment

\begin{tabular}{|c|c|c|c|c|c|c|}
\hline & \multicolumn{2}{|c|}{ Baseline $(N=24)$} & \multicolumn{2}{|c|}{ Round 1 (20 experiments) } & \multicolumn{2}{|c|}{ Other rounds (43 experiments) } \\
\hline & Mean & Std & Mean & Std & Mean & Std \\
\hline $\mathrm{pH}$ & 7.46 & 0.07 & 7.33 & 0.11 & 7.34 & 0.08 \\
\hline iCa $[\mathrm{mmol} / \mathrm{L}]^{*}$ & 1.28 & 0.09 & 1.23 & 0.08 & 1.19 & 0.12 \\
\hline
\end{tabular}

Improved $\mathrm{pH}$ between round 1 and other rounds are attributable to bicarbonate infusions during stabilization to prevent lethal acidemia

*One-way ANOVA between baseline, round 1, and other rounds demonstrated significant differences between the three groups $(p=0.03)$ 
Table 4 Results of blood chemistries from the 9 animals that received iCa in cohort 3

\begin{tabular}{|c|c|c|c|c|c|c|}
\hline & \multicolumn{2}{|c|}{ Baseline } & \multicolumn{2}{|c|}{ Round 1 ( $N=9$ rounds) } & \multicolumn{2}{|c|}{ Other Rounds ( $N=15$ rounds) } \\
\hline & Mean & Std & Mean & Std & Mean & Std \\
\hline $\mathrm{pH}$ & 7.45 & 0.024 & 7.22 & 0.14 & 7.31 & 0.077 \\
\hline PCO2 [mmHg] & 44.42 & 3.30 & 52.48 & 7.04 & 47.23 & 10.62 \\
\hline pO2 [mmHg] & 238.67 & 57.67 & 329.78 & 154.50 & 365.4 & 119.59 \\
\hline BEecf $[\mathrm{mmol} / \mathrm{L}]$ & 7 & 1.73 & -16 & 31.40 & -2.67 & 2.23 \\
\hline $\mathrm{HCO} 3[\mathrm{mmol} / \mathrm{L}]$ & 31.06 & 1.62 & 21.57 & 4.34 & 23.54 & 1.87 \\
\hline TCO2 [mmol/L] & 32.22 & 1.64 & 23.33 & 4.21 & 24.87 & 1.92 \\
\hline $\mathrm{sO} \%$ & 99.88 & 0.33 & 96.89 & 8.96 & 99.93 & 0.26 \\
\hline $\mathrm{Na}[\mathrm{mmol} / \mathrm{L}]$ & 137.55 & 1.88 & 140.22 & 9.90 & 139.87 & 2.26 \\
\hline $\mathrm{K}[\mathrm{mmol} / \mathrm{L}]$ & 4.2 & 0.23 & 3.79 & 0.43 & 4.12 & 0.62 \\
\hline iCa [mmol/L] & 1.3867 & 0.05 & 1.22 & 0.14 & 1.33 & 0.15 \\
\hline Glu [mg/dL] & 83.22 & 23.75 & 213.11 & 127.61 & 152 & 85.83 \\
\hline HCT [\% PCV] & 23.22 & 3.27 & 32.44 & 3.81 & 30.13 & 4.31 \\
\hline $\mathrm{HB}[\mathrm{g} / \mathrm{dl}]$ & 7.911 & 1.13 & 11.03 & 1.31 & 10.25 & 1.47 \\
\hline
\end{tabular}

*Improved base excess, pCO2, and pH between round 1, and other rounds are attributable to bicarbonate infusions during stabilization to prevent lethal acidemia

averaged as a function of time. The mean aortic pressure responses are represented by the blue line in Fig. 2. The shaded region above and below the blue line represents the standard deviation of those values. The mean pressures and the standard deviation of the pressures approaches zero as the time approaches zero due to the normalization procedure discussed in the methods. Comparing blood pressures from the timespan

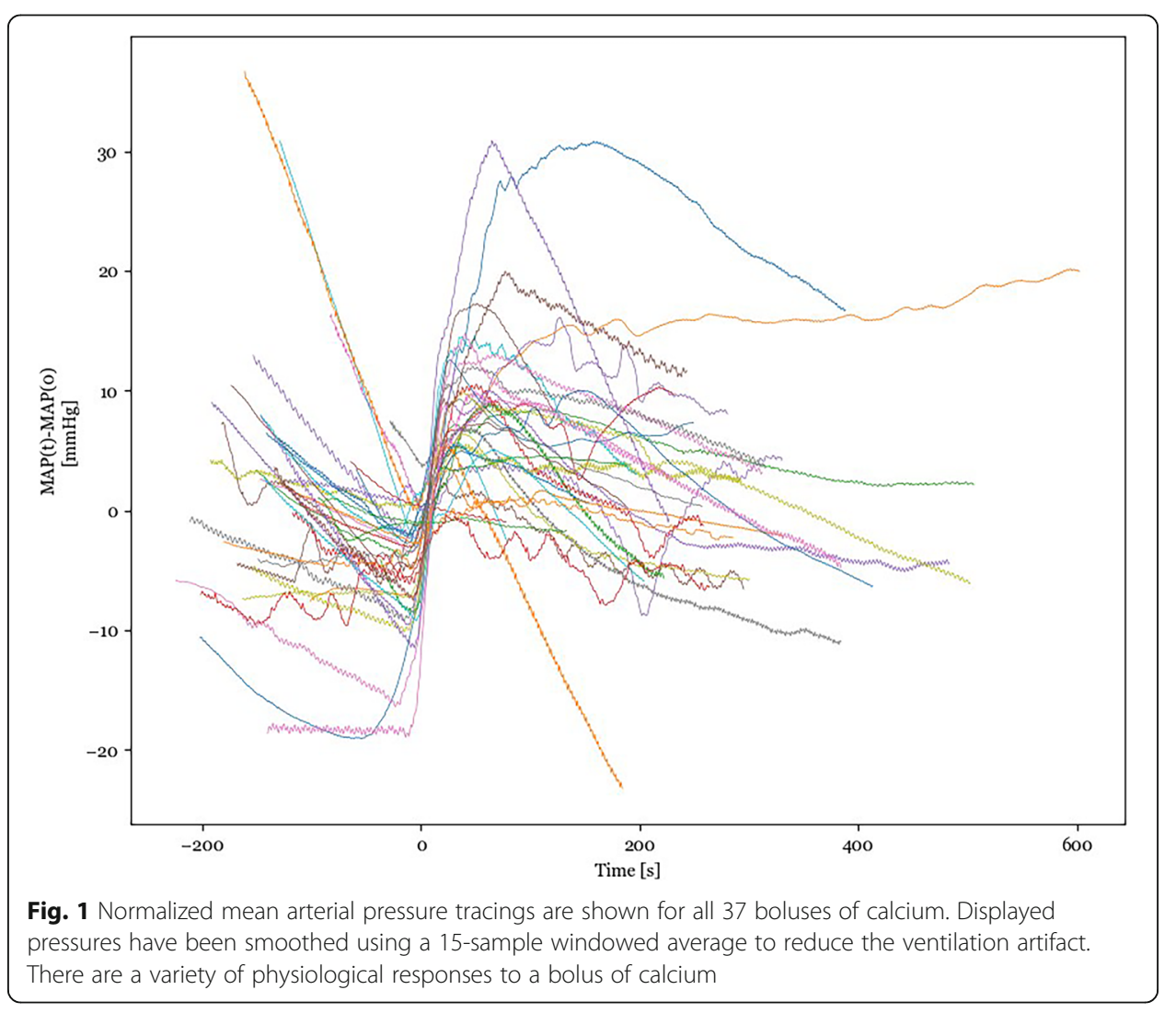




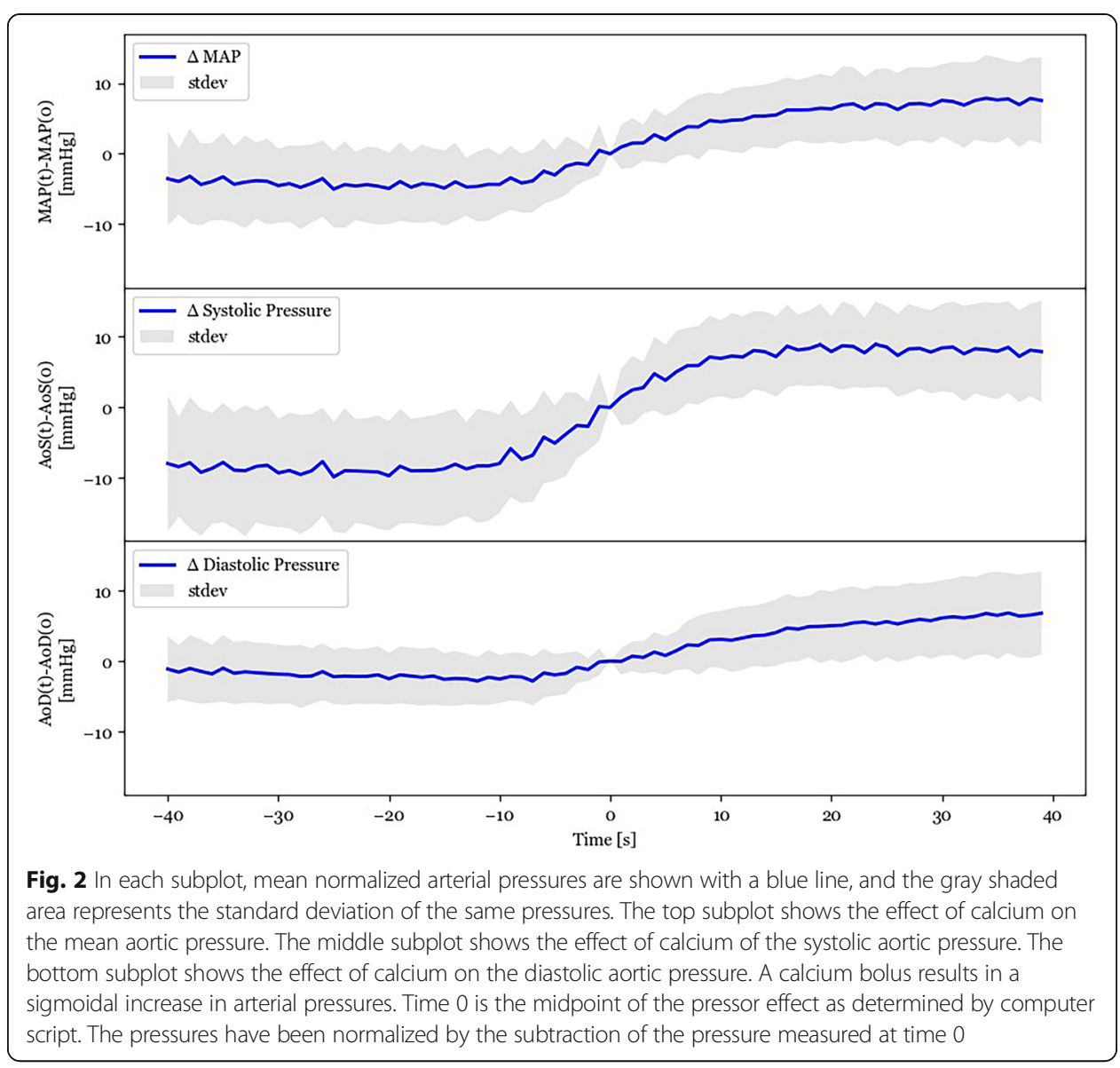

[ $-40 \mathrm{~s}<t<-36 \mathrm{~s}$ ] with blood pressures from the timespan [36 $\mathrm{s}<t<40 \mathrm{~s}$ ], we find that the MAP increased by $12.7 \pm 6.5 \mathrm{mmHg}(p<0.05)$, the AoS increased by $10.6 \pm 8.5$ $\mathrm{mmHg}(p<0.05)$, and the AoD increased by $3.1 \pm 4.5 \mathrm{mmHg}(p<0.05)$.

The physiological response to the calcium boluses manifested in a range of MAP responses, as shown in Fig. 3. In Fig. 4, the histogram shows the range of changes in the MAP for all 37 boluses. In four experiments, the MAP $40 \mathrm{~s}$ after the bolus was lower than the MAP $40 \mathrm{~s}$ before the bolus.

\section{Discussion}

Our preliminary results indicate that similar to ventricular fibrillation, ionized hypocalcemia may be common after resuscitation from pseudo-PEA, and the associated refractory hypotension may be responsive to administration of IV calcium. Specifically, administration of calcium in this setting is often associated temporally with a relatively rapid pressor response. Of note, calcium administration was also often associated with a stabilization of blood pressure when it had been previously trending downward. In 16 instances, this stabilization was maintained until the end of that cycle in the experiment. In the remainder of cases, bolus calcium either resulted in only a temporary pressure increase followed by a return to progressive decline or no measurable effect. In the four instances, where MAP $40 \mathrm{~s}$ after the bolus was lower than the MAP $40 \mathrm{~s}$ before, the MAP was decaying rapidly and the ongoing rapid loss of blood pressure was 


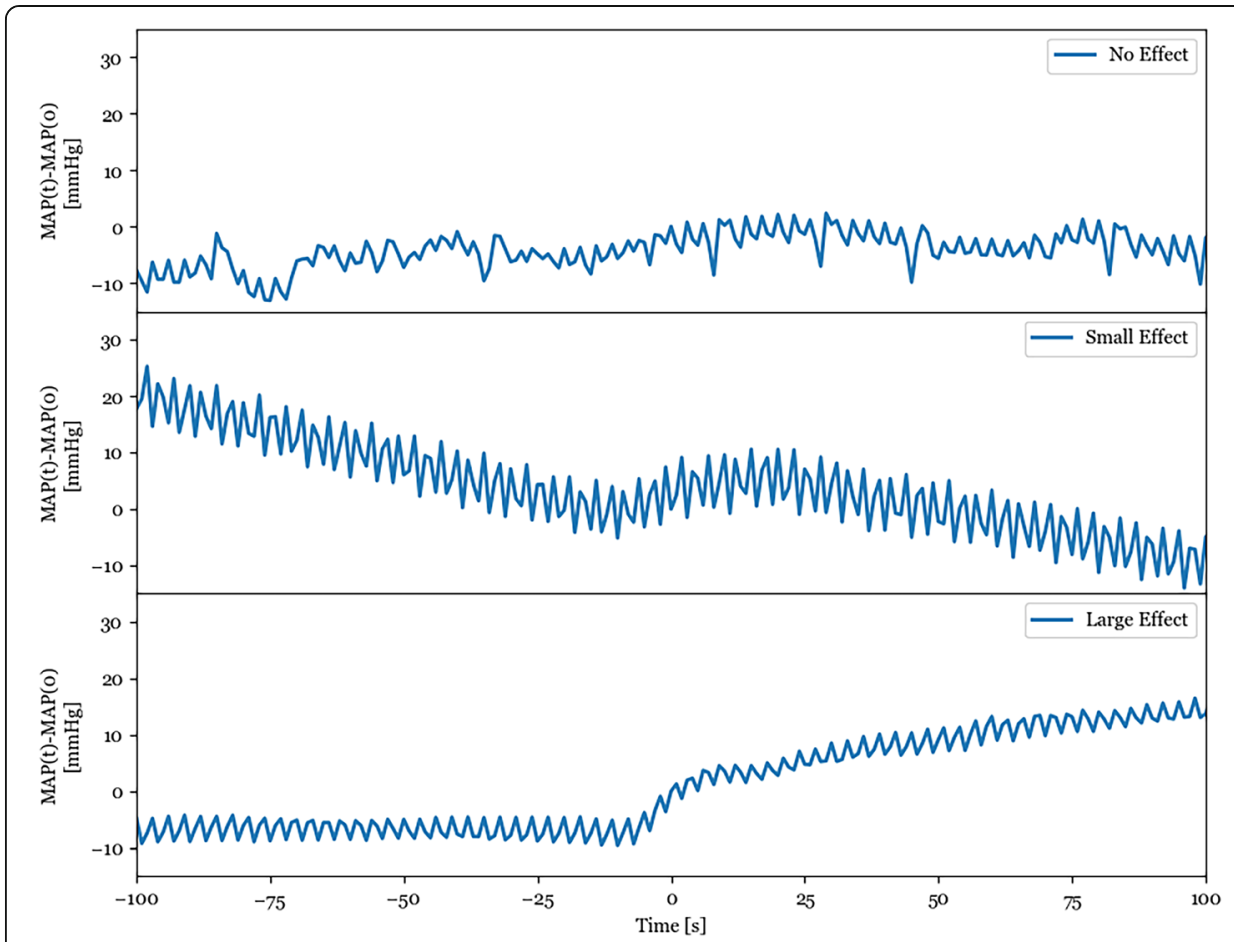

Fig. 3 There are a variety of individual responses to a calcium bolus. The top subplot shows a calcium injection that has no observable effect on the mean arterial pressure. The middle subplot shows a calcium injection that has a modest effect on the mean arterial pressure, but the MAP resumes its decline after about $20 \mathrm{~s}$ of improvement. The bottom subplot shows a calcium injection that has a large effect on arterial pressure where the MAP improves due to the calcium bolus and then continues to improve and stabilize as a function of time. These tracings were chosen to represent the spectrum of observed responses

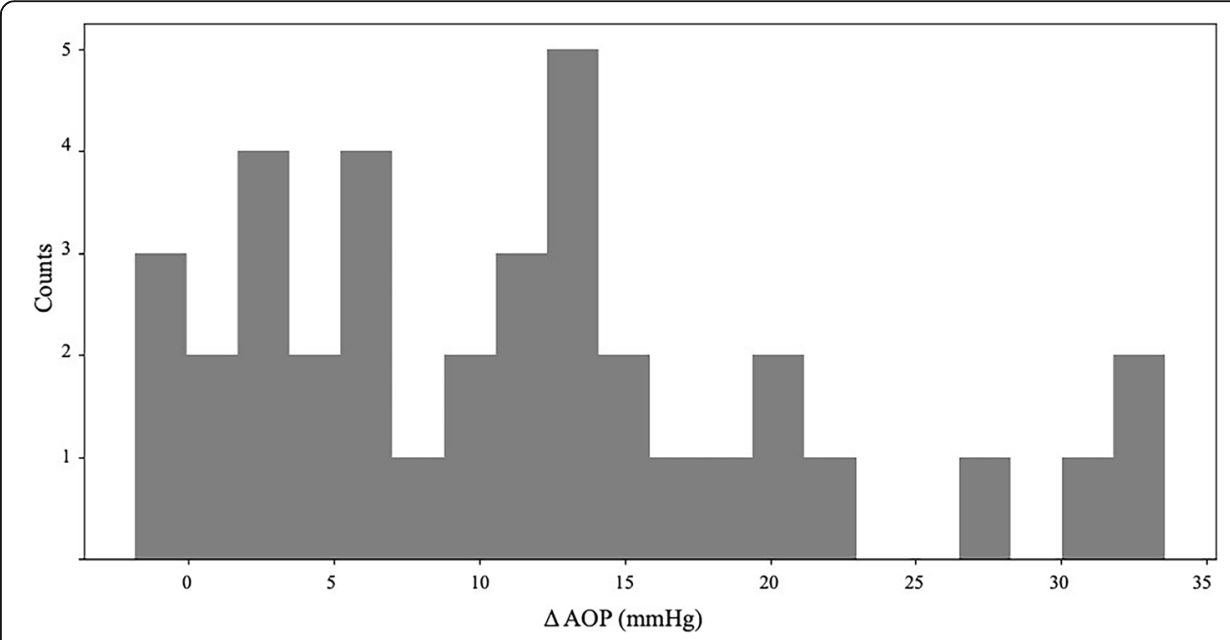

Fig. 4 This histogram of the change in mean aortic pressure was calculated by subtracting the mean aortic pressure $36-40 \mathrm{~s}$ before the calcium bolus from the mean aortic pressure $36-40 \mathrm{~s}$ after the calcium bolus. While most of these interventions are associated with a positive change in mean aortic pressure, in four experiments, the mean aortic pressure decreased. In these experiments, the animal was decompensating rapidly, and the continuing drop in blood pressure was larger than the benefit of the calcium bolus 
larger than the benefit of the calcium bolus, resulting in a negative pressure change. In these cases, it might be inaccurate to assume that the treatment effect resulted in a decrease in blood pressure or that the effect was marginal.

The relationship between ionized calcium and critical cardiovascular states is not fully understood [4]. Prior reports describe hypocalcemia in other cardiac arrest states [11]. Of the metabiolic abnormalities in these other hypotensive states, it was noted that there was often a relationship between ionized calcium concentration and $\mathrm{pH}$. Proposed explanations of this phenomena include intracellular calcium influx from ion compartment shifting, changes from impaired transmembrane pump activity, and extracellular protein complexing. Under ischemic conditions, it has been demonstrated that cells increase their uptake of calcium as a result of altered membrane transport; moreover, intracellular and mitochondrial calcium sequestration is an important initiator of apoptosis [12]. It is likely that a combination of these factors may contribute to an overall depletion in ionic calcium stores in pseudo-PEA. Ischemia and the resultant loss of normal cellular energy state seem likely to underlie these pathophysiologic processes.

Hypocalcemia likely also contributes to the hypotension that is common in the recovery period of pseudo-PEA. Both cardiac and vascular smooth muscles are dependent on calcium for function. In cardiac myocytes, calcium-dependent calcium release is a critical component initiating robust ventricular contraction; hypocalcemia is a known cause of negative inotropy and dysrhythmia. For vascular smooth muscle, calcium is needed to activate calmodulin to allow for fiber crosslinking. Effective calcium starvation impairs vasomotor tone and results in a decrease in peripheral resistance. It is thus not surprising that hypotension after pseudo-PEA is often refractory to catecholamine pressors. Functional calcium deficits in both the myocardium and vascular smooth muscle likely blunt the sympathetic stimulation. Diffuse hypoxic insult with associated metabolic derangement may underlie continued hypocalcemia even after ROSC is achieved.

Hypotension after resuscitation from profound global hypoxia and ischemia, as in our porcine model of pseudo-PEA, is a particularly challenging cardiovascular state for clinicians. Leaving it untreated may worsen outcome through secondary injury, as in a "two-hit" model. As noted before, it is often refractory to catecholamine pressers [1], which may themselves be associated with a worse outcome [12]. It was relatively common during the 1960s and 1970s to treat cardiac arrest with boluses of IV calcium without evidence of efficacy [13]. This has become much less common in the era of guideline-based advanced life support.

Pseudo-PEA is common clinically-possibly present in half of all arrests-and outcomes are often poor [14]. It has not been studied to the same extent as ventricular fibrillation. The pseudo-PEA cardiovascular state can be created by hypoxia, asphyxia, cardiotonic drug overdose, and post-defibrillation [15]. Unfortunately, it has been difficult to create stable models using these insults. Our model is relatively stable and reproducible. However, the hypoxic insult in juvenile pigs may be most representative clinically of pediatric partial asphyxiation [15], such as is seen in asthma, and not the PEA caused by coronary occlusion in adults. For this reason, the applicability of our model to adult patients suffering ischemic cardiac arrest may be limited. 
Although collected retrospectively, our preliminary data indicates that ionized hypocalcemia may be present after resuscitation from pseudo-PEA and that bolus IV calcium is associated with a sustained pressor response. Intriguingly, this pressor effect often stabilized progressively declining blood pressure. These finding should be investigated prospectively. If confirmed, they may justify a clinical trial of intravenous calcium in the setting of pseudo-PEA or hypotension after resuscitation from pseudo-PEA.

However, it should be emphasized that hypotension is a physiologic biomarker and not a clinical outcome per se. It is possible that administration of intravenous calcium temporarily improves blood pressure but then is associated with an overall worse clinical outcome because it enhances vital organ injury by priming of apoptosis [16]. It is important that clinicians not respond to this preliminary post hoc data set by empiric administration of IV calcium to patients suffering pseudo-PEA. A clinical trial with a primary endpoint of improved neurologically intact long-term survival would need to be completed preliminary to a change in clinical practice.

Limitations-our report has a number of limitations: (1) this is an animal model of hypoxic pseudo-PEA, (2) the porcine model is juvenile and without senescent comorbidities, (3) intravenous calcium levels and the blood pressure response to calcium administration were not the primary processes under study, these are retrospectively observed results, (4) the before/after comparison used does not necessarily capture the effect of the calcium bolus, as in the four cases where MAP decreased due to rapid decompensation, (5) improved MAP is not a clinical outcome.

\section{Conclusion}

Our preliminary results indicate that the hypotension after hypoxia-induced pseudoPEA is frequently associated with decreased serum ionized calcium levels and a hypotension that is responsive to administration of IV calcium. These results should be confirmed prospectively before consideration is given to clinical trials.

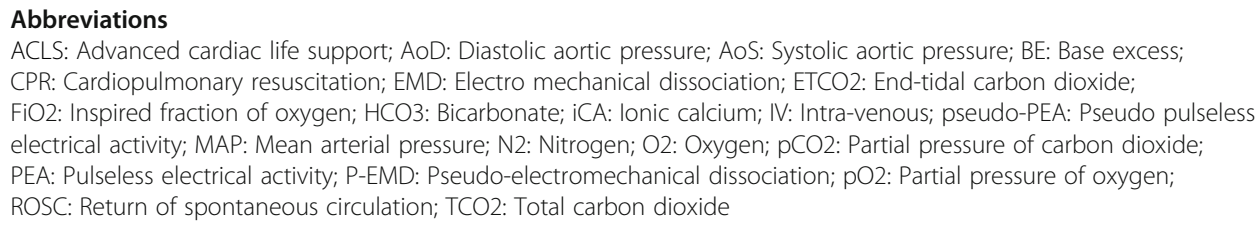

\section{Authors' contributions}

ALL conducted experiments and wrote the manuscript. JWL analyzed the data and was a major contributor in writing the manuscript. JRG and CLK designed the study and conducted the experiments. KM conducted the experiments. NAP designed the study, conducted the experiments, and was a major contributor in writing the manuscript. All authors consent to the publication of the manuscript. The author(s) read and approved the final manuscript.

Funding

The authors gratefully acknowledge funding from ZOLL Medical Corporation.

Availability of data and materials

The authors declare that all data supporting the findings of this study are available within the article. 
Consent for publication

Not applicable

\section{Competing interests}

The authors declare that they do not have any competing interests.

\section{Author details}

${ }^{1}$ Geisel School of Medicine, Dartmouth College, Hanover, NH 03775, USA. ${ }^{2}$ ZOLL Medical Corp, Chelmsford, MA 01824, USA. ${ }^{3}$ Geisel School of Medicine, Dartmouth Hitchcock Medical Center, 1 Medical Center Dr \#4B, Lebanon, NH 03756, USA.

Received: 8 April 2020 Accepted: 17 August 2020

Published online: 04 September 2020

\section{References}

1. Trzeciak S, Jones AE, Kilgannon JH et al (2009) Significance of arterial hypotension after resuscitation from cardiac arrest. Crit Care Med 37:2895-2903 quiz 904

2. Cairns CB, Niemann JT, Pelikan PC, Sharma J (1991) lonized hypocalcemia during prolonged cardiac arrest and closedchest CPR in a canine model. Ann Emerg Med 20:1178-1182

3. Niemann JT, Adomian GE, Garner D, Rosborough JP (1985) Endocardial and transcutaneous cardiac pacing, calcium chloride, and epinephrine in postcountershock asystole and bradycardias. Crit Care Med 13:699-704

4. Erdmann E, Reuschel-Janetschek E (1991) Calcium for resuscitation? Br J Anaesth 67:178-184

5. Paradis NA, Martin GB, Goetting MG, Rivers EP, Feingold M, Nowak RM (1992) Aortic pressure during human cardiac arrest. Identification of pseudo-electromechanical dissociation. Chest 101:123-128

6. Paradis NA, Halperin HR, Zviman M, Barash D, Quan W, Freeman G (2012) Coronary perfusion pressure during external chest compression in pseudo-EMD, comparison of systolic versus diastolic synchronization. Resuscitation 83:1287-1291

7. Jentzer JC, Chonde MD, Dezfulian C (2015) Myocardial dysfunction and shock after cardiac arrest. Biomed Res Int 2015: 314796

8. Paradis NA, Crockett SC, Moodie K, Gould JR (2017) Relationship between right atrial pressure with jugular flow in an asphyxial model of pseudo-pulseless electrical activity in swine. Circulation 136:A15994

9. Paradis NA, Crockett SC, Gould JR, Kaufman C, Moodie K (2018) Development of a hypoxic asphyxial model of pseudopulseless electrical activity. Circulation 136:A15914

10. Larabee TM, Paradis NA, Bartsch J, Cheng L, Little C (2008) A swine model of pseudo-pulseless electrical activity induced by partial asphyxiation. Resuscitation 78:196-199

11. Yang L, Weil MH, Noc M, Tang WC, Turner T, Gazmuri RJ (1994) Spontaneous gasping increases the ability to resuscitate during experimental cardiopulmonary resuscitation. Crit Care Med 22:879-883

12. Callaham ML, Barton CW, Kayser S (1991) Potential complications of high-dose epinephrine therapy in patients resuscitated from cardiac arrest [see comments]. JAMA 265:1117-1122

13. Donovan PJ, Propp DA (1985) Calcium and its role in cardiac arrest: understanding the controversy. J Emerg Med 3:105116

14. Andrew E, Nehme Z, Lijovic M, Bernard S, Smith K (2014) Outcomes following out-of-hospital cardiac arrest with an initial cardiac rhythm of asystole or pulseless electrical activity in Victoria, Australia. Resuscitation 85:1633-1639

15. Newman J (2013) Pulseless electrical activity in a pediatric patient: a case report and review of causative factors and treatment. AANA J 81:459-464

16. Cerella C, Diederich $M, \&$ Ghibelli $L$ (2010). The Dual Role of Calcium as Messenger and Stressor in Cell Damage, Death, and Survival. International.Journal of Cell Biology, 2010, 1-14. https://doi.org/10.1155/2010/546163.

\section{Publisher's Note}

Springer Nature remains neutral with regard to jurisdictional claims in published maps and institutional affiliations.

\section{Submit your manuscript to a SpringerOpen ${ }^{\circ}$ journal and benefit from:}

- Convenient online submission

- Rigorous peer review

- Open access: articles freely available online

- High visibility within the field

- Retaining the copyright to your article

Submit your next manuscript at $\boldsymbol{\nabla}$ springeropen.com 